\title{
CRISPR/Cas9-mediated targeted mutation reveals a role for $A N 4$ rather than DPL in regulating venation formation in the corolla tube of Petunia hybrida
}

\author{
Bin Zhang ${ }^{1,2}$, Xiaojing X X ${ }^{1}$, Renwei Huang ${ }^{1,3}$, Sha Yang ${ }^{1}$, Mingyang $\mathrm{Li}^{1}$ and Yulong Guo ${ }^{1}$
}

\begin{abstract}
Venation is a common anthocyanin pattern displayed in flowers that confers important ornamental traits to plants. An anthocyanin-related R2R3-MYB transcription factor, DPL, has been proposed to regulate corolla tube venation in petunia plants. Here, however, we provide evidence redefining the role of DPL in petunia. A CRISPR/Cas9-mediated mutation of DPL resulted in the absence of the vein-associated anthocyanin pattern above the abaxial surface of the flower bud, but not corolla tube venation, thus indicating that DPL did not regulate the formation of corolla tube venation. Alternately, quantitative real-time PCR analysis demonstrated that the spatiotemporal expression pattern of another R2R3-MYB gene, AN4, coincided with the formation of corolla tube venation in petunia. Furthermore, overexpression of AN4 promoted anthocyanin accumulation by increasing the expression of anthocyanin biosynthesis genes. CRISPR/Cas9-mediated mutation of AN4 led to an absence of corolla tube venation, suggesting that this gene in fact determines this key plant trait. Taken together, the results presented here redefine the prime regulator of corolla tube venation, paving the way for further studies on the molecular mechanisms underlying the various venation patterns in petunia.
\end{abstract}

\section{Introduction}

Anthocyanin pigments contribute to the diverse colors and pigmentation patterns of flowers, which are important ornamental characteristics of horticultural plants. Anthocyanins are derived from the flavonoid biosynthetic pathway, involving a series of enzymes encoded by several structural genes ${ }^{1}$. In dicot plants, these structural genes can be subdivided into early biosynthetic genes (EBGs), including chalcone synthase (CHS), chalcone isomerase $(C H I)$, flavanone 3-hydroxylase (F3H), and flavonoid 3'hydroxylase $\left(F 3^{\prime} H\right)$, and late biosynthetic genes (LBGs), such as flavonoid $3^{\prime} 5^{\prime}$-hydroxylase $\left(F 3^{\prime} 5^{\prime} H\right)$, dihydroflavonol

\footnotetext{
Correspondence: Mingyang Li (limy@swu.edu.cn) or Yulong Guo (yulong@swu. edu.cn)

${ }^{1}$ Chongqing Engineering Research Center for Floriculture, Key Laboratory of Horticulture Science for Southern Mountainous Regions, Ministry of Education, College of Horticulture and Landscape Architecture, Southwest University, 400716 Chongqing, China

${ }^{2}$ College of Agriculture, Guizhou University, 550025 Guiyang, Guizhou, China Full list of author information is available at the end of the article
}

4-reductase (DFR), anthocyanidin synthase (ANS), and glutathione-S-transferase $(G S T)^{2-5}$. Expression of these EBGs is regulated by R2R3-MYB transcription factors ${ }^{6,7}$, while the expression of the LBGs is regulated by the ternary complex MYB-bHLH-WD40 consisting of R2R3-MYB, a basic helix-loop-helix (bHLH), and WD40 transcription factors $^{8,9}$. Among these transcription factors, R2R3-MYB genes play major roles in providing the required specificity for LBG expression and consequently determining the spatiotemporal accumulation of anthocyanin in plants ${ }^{10,11}$.

Venation patterning arises from pigmented stripes overlying veins and is a common anthocyanin pattern seen in flowers ${ }^{12}$. In Antirrhinum, the bHLH gene Delila is expressed in its petal epidermis, and Venosa, an R2R3MYB gene, is specifically expressed in cells above the vascular tissues, with venation arising in the overlapping expression domains of the R2R3-MYB and bHLH genes ${ }^{13}$. Since the bHLH and WD40 genes are usually expressed constitutively, the formation of venation patterning

\section{(c) The Author(s) 2021}

(c) (i) Open Access This article is licensed under a Creative Commons Attribution 4.0 International License, which permits use, sharing, adaptation, distribution and reproduction cc) in any medium or format, as long as you give appropriate credit to the original author(s) and the source, provide a link to the Creative Commons license, and indicate if changes were made. The images or other third party material in this article are included in the article's Creative Commons license, unless indicated otherwise in a credit line to the material. If material is not included in the article's Creative Commons license and your intended use is not permitted by statutory regulation or exceeds the permitted use, you will need to obtain permission directly from the copyright holder. To view a copy of this license, visit http://creativecommons.org/licenses/by/4.0/. 

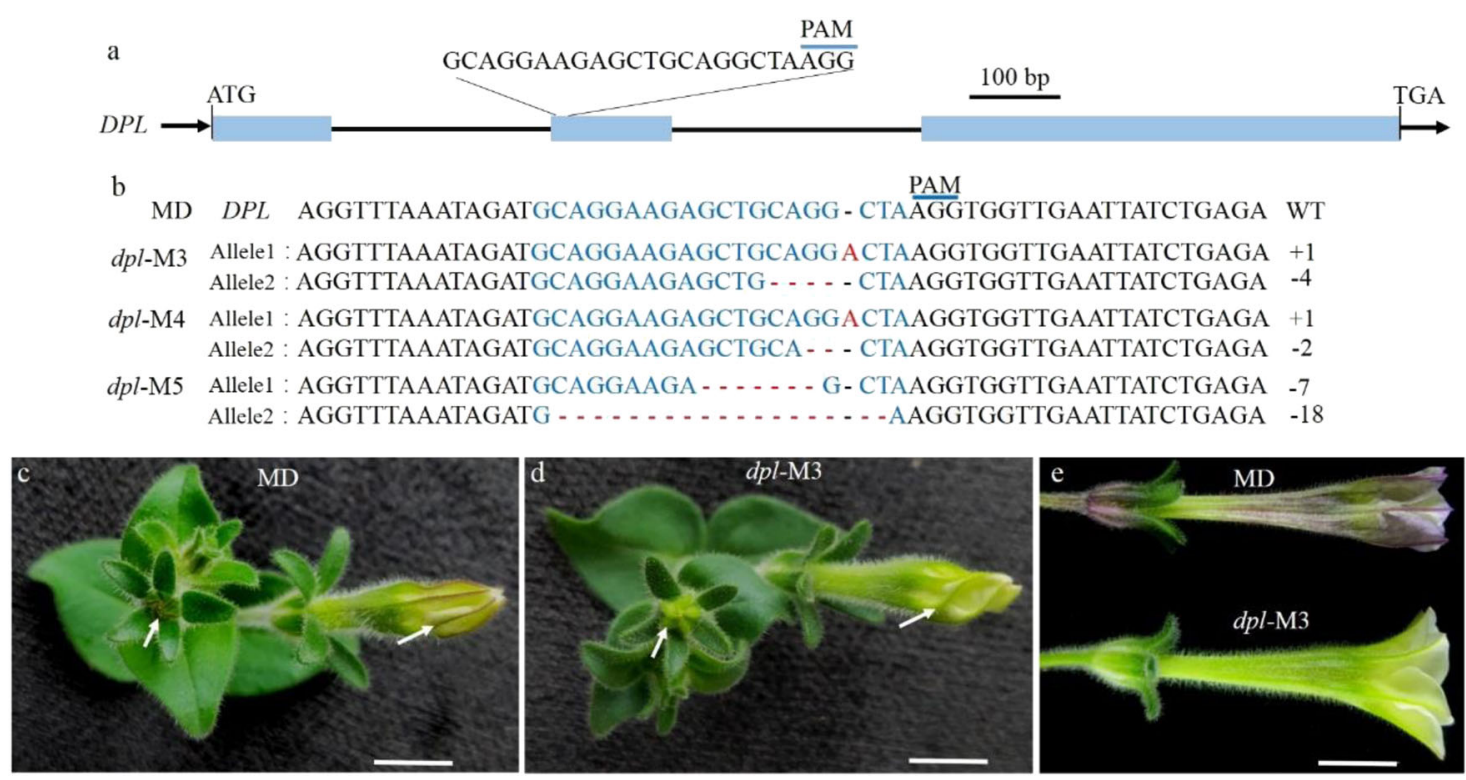

Fig. 1 CRISPR/Cas9-mediated mutation in DPL. a Diagram of the target site in the genomic region of DPL. The blue line indicates the PAM (NGG) motif. The blue boxes and black lines indicate exons and introns, respectively. $\mathbf{b}$ Targeted mutagenesis in DPL for the dpl-M3, -M4, and -M5 lines. The target sequence is shown in blue letters, for which red dashes indicate deletions, while red letters indicate insertions. c-e Flower buds of MD and a representative line $\mathrm{dp} / \mathrm{M} 3$; flower buds in $\mathbf{c}$ and $\mathbf{d}$ are from plants cultured at $25^{\circ} \mathrm{C}$, and those in e are from plants treated at $12 \pm 2{ }^{\circ} \mathrm{C}$ (cold stress conditions). Scale bars $=1 \mathrm{~cm}$

depends mainly on $R 2 R 3-M Y B$, which provides vein specificity. For example, in venosa mutants, no venation is displayed in their petals ${ }^{14}$, and silencing of PeMYB12, an R2R3-MYB gene, induces the loss of venation patterning in sepals/petals of Phalaenopsis spp. ${ }^{15}$. In addition to venation, R2R3-MYB is also the key regulator of two other anthocyanin patterns: blotch $^{16}$ and spot ${ }^{17,18}$.

Petunia hybrida, distinguished by its diverse flower colors and pigmentation patterns, is one of the most popular bedding plants ${ }^{19}$. In such petunia plants, diverse types of venation patterning have been developed in the corolla of some cultivars to improve their ornamental value. Several genetic loci reportedly control the various patterns of venation in the corolla of petunias. For example, individuals harboring the Venation-1 (Ve1) locus display reticulate venation of the corolla tube, whereas ve1 individuals exhibit a few longitudinal pigmented stripes over the veins; Venation-2 (Ve2) and Venation-3 (Ve3) loci determine the extension of venation from the corolla throat to the outer edge of the limb; and the Fine venation $(F n)$ locus controls the thickness of venation in the corolla $\operatorname{limb}^{20}$. A previous study proposed that an anthocyaninrelated R2R3-MYB gene, DEEP PURPLE (DPL), may reside at the $V e 1$ locus $^{21}$. The role of $D P L$ in the regulation of venation patterning in corolla tubes has since been cited widely in many recent publications ${ }^{12,15,22-24}$. In addition to $D P L$, another anthocyanin-related R2R3-MYB gene, ANTHOCYANIN4 (AN4), is capable of associating with these $V e$ and $F n$ loci according to genetic analyses. For example, a stronger venation is associated with the presence of the dominant allele of $A N 4$ and the recessive ve2 allele $^{20,25}$. We know that $A N 4$ can regulate the anthocyanin accumulation in anthers ${ }^{26}$, but whether this gene also determines venation patterning remains unknown.

In this work, we reinvestigated the respective roles of $D P L$ and AN4 in flower development, mainly by using CRISPR/Cas9 targeted mutation technology. We found that venation patterning in petunia corolla tubes is actually regulated by $A N 4$, not the proposed $D P L$. Our results also revealed that $D P L$ 's true role in petunia flower petals is to regulate their vein-associated anthocyanin patterns above the abaxial surface.

\section{Results}

\section{CRISPR/Cas9-induced mutation of DPL in MD}

To investigate the specific role of $D P L$ in petunia, we mutated DPL in the P. hybrida inbred line "Mitchell Diploid' (MD) by using the CRISPR/Cas9 system. One target site in the second exon was selected as the sgRNA complementary site (Fig. 1a), and three DPL biallelic mutants (dpl-M3, -M4, and -M5) in MD were obtained. In the $d p l$-M5 line, one allele of $D P L$ exhibited a deletion of $18 \mathrm{bp}$, which introduced a new stop codon in DPL, leading to premature terminations. The deletion in the second $D P L$ allele of $d p l$-M5 and the indel mutations in the DPL alleles of $d p l-\mathrm{M} 3$ and dpl-M4 mutants interrupted the 


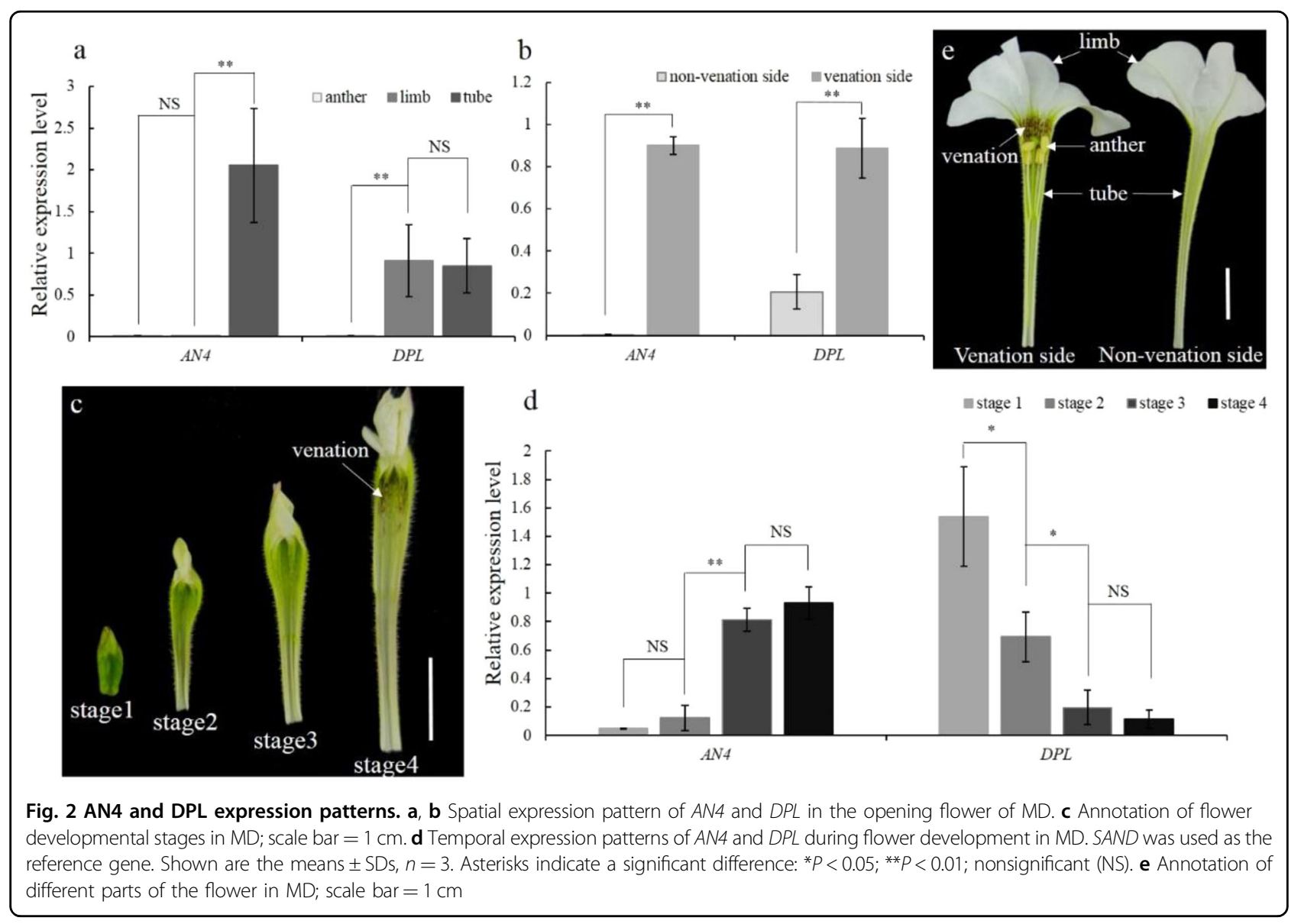

reading frame, leading to frameshift mutations (Fig. 1b). Considering that the target sequence of $D P L$ is highly similar to that in $A N 4 I$ and $A N 4 I I$, a potential off-target site was detected in AN4I and AN4II of the dpl-M3, -M4, and -M5 lines; however, no mutations could be found in the off-target site of these lines (Fig. S3a). Therefore, $d p l$ $\mathrm{M} 3$, -M4, and -M5 were $d p l$-knockout mutants and were used for further investigations.

No significant changes in corolla tube venation patterning were detected between $d p l$ mutants and MD (Fig. S1a-d). However, vein-associated anthocyanin deposition above the abaxial surface of the flower bud was lost in $d p l$ mutants (Fig. 1c, $\mathrm{d}$ and Fig. S1e-g). In the T1 lines of $d p l$ M3, -M4, and -M5, this mutated phenotype was observed. Vein-associated anthocyanin patterning initially emerged at the flower bud abaxial surface, but gradually faded as the flower opened (Fig. S1h). Furthermore, this anthocyanin patterning became more obvious in flower buds of $\mathrm{MD}$, when these plants with flower buds were treated at $12 \pm 2{ }^{\circ} \mathrm{C}$ for 2 weeks, but it was always absent in those of $d p l$-knockout mutants (Fig. 1e). These results indicated that $D P L$ determines vein-associated anthocyanin occurrence above the abaxial epidermis of petunia flower buds rather than the venation patterning of its corolla tube.
Positive correlation between the expression patterns of AN4 and the formation of corolla tube venation

Because venation patterning was not affected in $d p l$ mutants, we next investigated whether other MYB genes were perhaps involved in regulating petunia venation patterning. qPCR analysis showed that the mRNA abundance of AN4 was much higher in the corolla tube than in the anther or limb, whereas the mRNA abundance of $D P L$ was much higher in the limb and corolla tube than in the anther (Fig. 2a). Notably, within the corolla tube, the transcript level of $A N 4$ was higher on the venation side than on the nonvenation side after the opening of the flower (Fig. 2b). The transcript level of $D P L$ was higher on the venation side than on the nonvenation side and was higher than the expression of AN4 on the nonvenation side (Fig. 2b).

Going further, we then investigated the $A N 4$ and $D P L$ expression levels during the flower development. Based on corolla tube length $(0.5,1.5,2.5$, and $3.5 \mathrm{~cm})$, flower development was divided into stage 1 (S1), stage 2 (S2), stage 3 (S3), and stage 4 (S4; Fig. 2c). During flower development, the venation pattern was gradually accentuated by the presence of anthocyanin. The mRNA abundance of $D P L$ continuously decreased from S1 to S4 
(Fig. 2d). However, the expression level of AN4 continuously increased from S1 to S4 (Fig. 2d), in accordance with the emergence of venation. These results indicated that AN4 could determine corolla tube venation in MD.

\section{AN4 promotes anthocyanin biosynthesis in MD}

To confirm that $A N 4^{M D}$ encoded a functional R2R3MYB transcription factor in MD, the transcript of $A N 4$ was isolated from this plant's corolla tube. Sequence analysis revealed that the open reading frame of $A N 4^{M D}$ was highly similar to that of $A N 4^{V 3 O}$, which has been identified as an activator of anthocyanin biosynthesis in the V30 line ${ }^{27}$. The deduced amino acid sequence of $A N 4^{M D}$, along with other anthocyanin-related R2R3MYBs in petunia, contained the conserved [D/E] $\mathrm{Lx}_{2}[\mathrm{R} / \mathrm{K}]$ $\mathrm{x}_{3} \mathrm{Lx}_{6} \mathrm{Lx}_{3} \mathrm{R}$ motif in its $\mathrm{R} 3$ domain (Fig. 3), as required for interacting with the AN1 (bHLH) protein ${ }^{21}$. Outside the conserved R2 and R3 domains, several substitutions of amino acids were present in $A N 4^{M D}$ compared with $A N 4^{V 30}$ (Fig. 3).

To verify the function of $A N 4^{M D}$ in $\mathrm{MD}$, transgenic lines expressing $A N 4^{M D}$ under the control of the CaMV35S promoter were generated in the MD background. Twelve independent transgenic lines (AN4OE) with pigmented leaves, stems, anthers, and corolla limbs were obtained (Fig. 4a, b). Although anthocyanins had accumulated in the anthers of these transgenic lines, their pollen was still yellow (Fig. 4c). The mRNA abundance of AN4 in the five transgenic lines was assessed by qPCR. As expected, the transcription of AN4 in these lines was higher than that in $\mathrm{MD}$, and correspondingly, their anthocyanin content was markedly increased (Fig. S2a, b). We next investigated the effect of overexpressing $A N 4^{M D}$ on the mRNA abundance of anthocyanin biosynthetic genes. These qPCR results revealed that the expression levels of most of these genes, including CHSA, CHSI, F3H, $F 3^{\prime} H, F 3^{\prime} 5^{\prime} H, D F R, A N S, 3 R T, 5 G T$, and GST but not $F 3^{\prime}$ $H$, were strongly upregulated in $A N 4 \mathrm{OE}$ transgenic plants compared with those in wild-type plants (Fig. 4d). These results indicated that $\mathrm{AN} 4^{\mathrm{MD}}$ functions as a transcriptional activator of the anthocyanin biosynthetic pathway in MD.

\section{AN4 determines corolla tube venation patterning}

To identify the function of $A N 4$ in regulating corolla tube venation, the CRISPR/Cas9 system was applied to generate an AN4 mutant. Referring to the genome sequence of Petunia axillaris ${ }^{19}$, two AN4 genes, AN4I and AN4II, containing the same gene structure but different promoters were isolated from the MD genome (Fig. 5a). One sgRNA was designed to simultaneously target the second exon of both AN4I and AN4II (Fig. 5a); in this way, seven transgenic lines without venation patterning in their corolla tube were obtained. Three nonvenation lines (an4-M1, -M2,

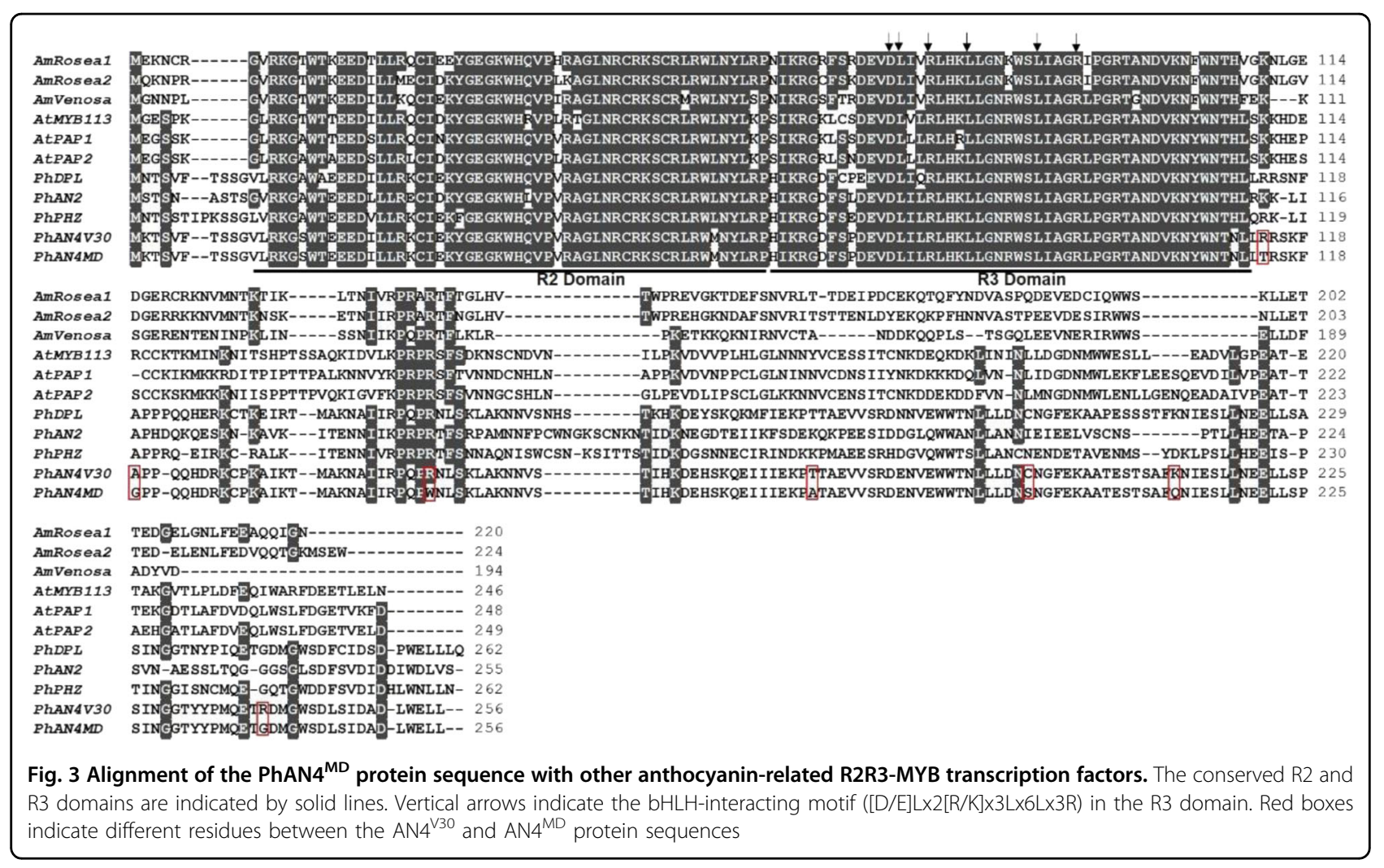




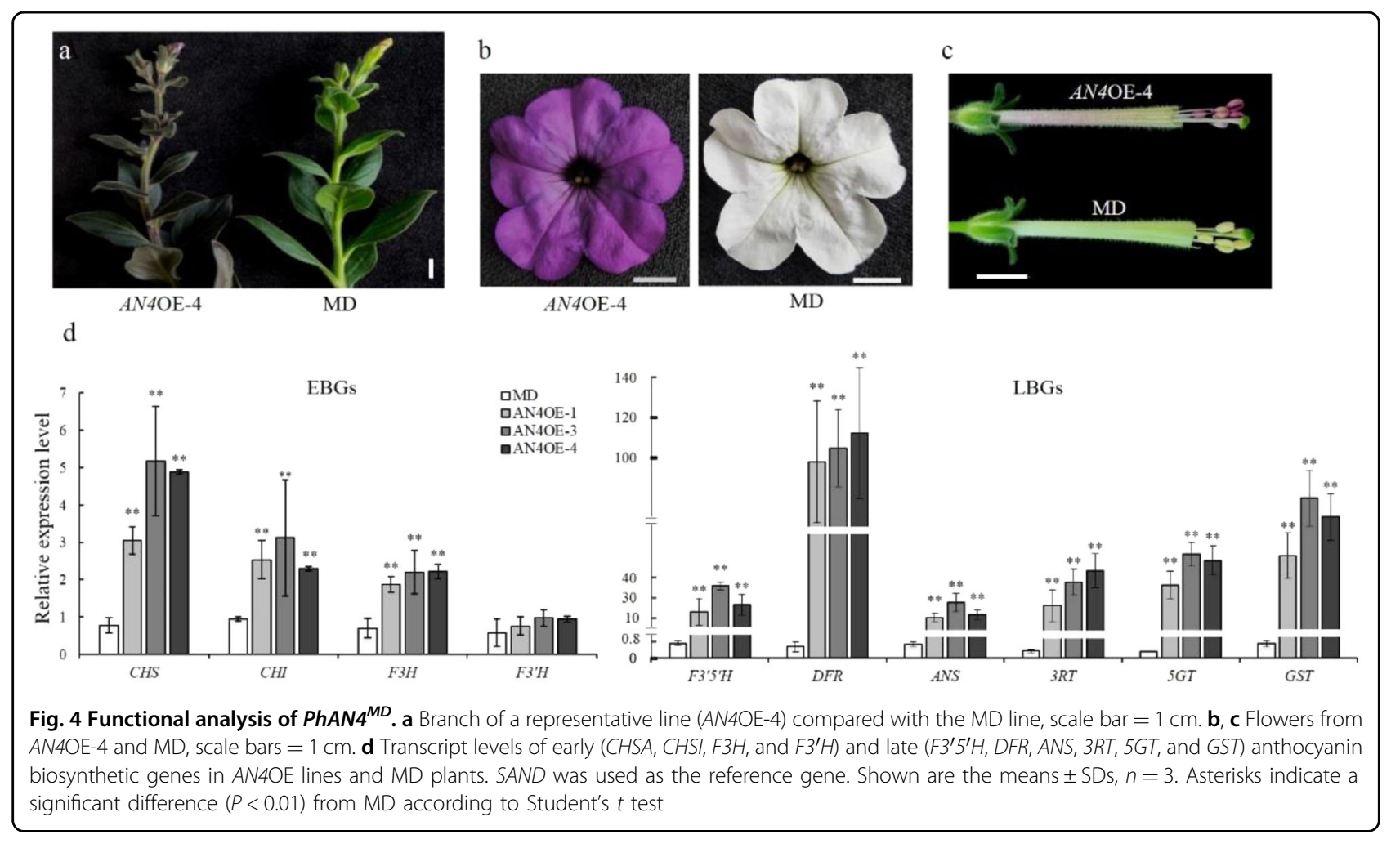

and -M6) were randomly selected to detect the mutation types in the AN4I and AN4II loci. In the an4-M1 and an4M6 lines, biallelic mutations occurred in the AN4I and AN4II loci, and the an4-M2 line harbored a biallelic mutation of AN4II and a monoallelic mutation of AN4I (Fig. 5b). The indel mutations in these mutants resulted in frameshift mutations, rendering the AN4 protein inactive. In the T1 lines of an4-M1, -M2, and -M6, venation patterning disappeared in the corolla tube as well. Considering that the target sequences of AN4 and DPL were highly similar to each other, a potential off-target site was detected in the DPL locus of the an4-M1, -M2, and -M6 lines; however, no mutations could be found in the off-target site of these lines (Fig. S3b).

To investigate the role of AN4 in regulating the expression of EBGs and LBGs, qPCR was carried out to detect the relative expression levels of anthocyanin biosynthetic genes in the an4-M1, -M2, and -M6 lines. These results showed that transcript levels of EBGs (CHSA, $C H S I, F 3 H$, and $F 3^{\prime} H$ ) went unchanged, but those of LBGs $\left(F 3^{\prime} 5^{\prime} H, D F R, A N S, 3 R T, 5 G T\right.$, and GST) were significantly downregulated in the an4-M1, -M2, and -M6 lines compared with those in MD (Fig. 5c). Corresponding to the nonvenation phenotype, the anthocyanin content was dramatically reduced in the an4-M1, -M2, and -M6 lines compared with that in MD (Fig. S2c). These results indicated that $A N 4$ preferentially governs LBGs to control the pigmentation of corolla tube venation.

\section{Discussion}

Petunia is a classical model system for studying the biosynthesis and regulation of flower pigments ${ }^{28-30}$. Some pigmentation patterns in petunia flowers have been well characterized. For example, the spot pattern is the result of an unstable transposon insertion in the ANTHOCYANIN3 locus $^{31}$, and star-type and picotee patterns are induced by RNA silencing of $C H S^{32,33}$. In this paper, we revealed that venation patterning in the corolla tube is determined by the R2R3-MYB transcription factor AN4, but not DPL.

Formerly, according to patterns of gene expression, segregation analysis, and vein-associated promoter activity, $D P L$ was proposed to govern venation patterning in MD flower tubes ${ }^{21}$. However, the lines of evidence for this were indirect and inconclusive. First, the mRNA abundance of $D P L$ gradually decreased during flower development (Fig. $2 \mathrm{~d})$, coinciding with the disappearance of vein-associated anthocyanin on the abaxial epidermis of the flower bud (Fig. S1h), whereas the venation patterning gradually emerged during flower development, coinciding with AN4 expression (Fig. 2d). Second, despite the cosegregation of $D P L$ and venation patterning, the latter's formation may have been driven by $A N 4$ instead of $D P L$ in the segregation analysis because $A N 4$ and DPL are on the same chromosome, lying just $60 \mathrm{~kb}$ apart ${ }^{19}$. Third, loss-of-function mutants or gene-silenced lines of $D P L$, which are important for verifying the specific roles of $D P L$ in petunia, were lacking. Hence, overall, $D P L$ might not determine venation 


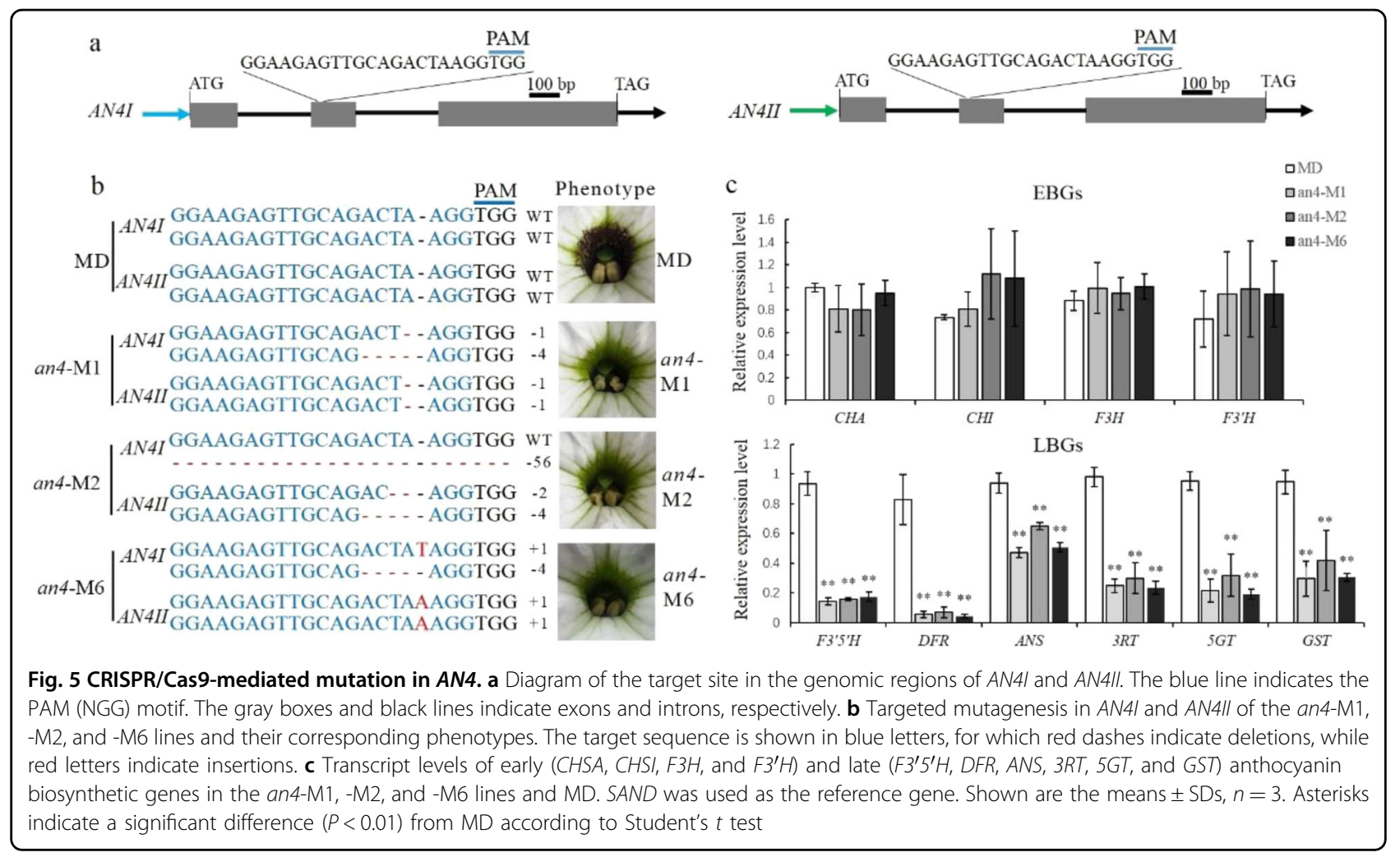

patterning in petunia. By relying on the CRISPR/ Cas9 system, we were able to obtain sought-after $d p l$ mutants. In these, vein-associated anthocyanins on the abaxial epidermis of flower buds disappeared rather than venation patterning, which unchanged (Figs. 1d and S1). Combining the vein-associated promoter activity of $D P L^{21}$ and the phenotype of CRISPR/Cas9-induced DPL mutants suggests that $D P L$ 's role is to determine vein-associated anthocyanin patterning on the abaxial epidermis of flower buds. Nonetheless, vein-associated anthocyanin patterning was more pronounced under cold conditions (Fig. 1e) and matched well to DPLpro-directed GUS localization in the corolla $^{21}$, thus suggesting that DPL may respond to cold stress in petunia.

To date, it has been reported that nine MYB genesAN4, AN2, DPL, PURPLE HAZE (PHZ), MYB27, MYBX, ANTHOCYANIN SYNTHESIS REGULATOR 1 (ASR1), $A S R 2$, and ASR3-regulate anthocyanin biosynthesis in petunia $^{21,23,26,34,35}$. These genes act as transcriptional activators or repressors of anthocyanin biosynthesis by positively or negatively regulating the expression of anthocyanin biosynthetic genes. Previous research has demonstrated that AN4 determines the pigmentation of anthers in petunia, so $A N 4$ is primarily regarded as having recessive alleles in some petunia lines with acyanic anthers, such as those of MD, V26, R27, and W138 (refs. ${ }^{26,28}$ ). However, we found that AN4 is a functional activator of anthocyanin biosynthesis genes in MD plants.
The expression levels of most EBGs were upregulated in transgenic plants overexpressing AN4 (Fig. 4d), but were not affected in CRISPR/Cas9-induced an 4 mutants (Fig. 5c). Similarly, the transcript levels of EBGs were not changed in the an 2 mutant $^{36}$. In Arabidopsis, EBGs are activated by flavonol-specific R2R3-MYBs, including MYB11, MYB12, and MYB111 (refs. ${ }^{37,38}$ ). The expression of EBGs in the an2 mutant and CRISPR/Cas9-induced an4 mutants might be activated by the orthologs of AtMYB11, AtMYB12, and AtMYB111, which have not been isolated in petunia. Therefore, it remains challenging to improve the regulatory mechanism controlling the expression of EBGs and LBGs in petunias.

During flower development, the spatiotemporal expression pattern of AN4 was positively associated with the formation of venation patterning in the corolla tube (Fig. 2a, b, d). Furthermore, CRISPR/Cas9-induced AN4 mutation resulted in the complete absence of venation patterning in MD corolla tubes (Fig. 5b). Together, these results indicate that $A N 4$ determines venation patterning in the corolla tube of MD flowers. In addition to regulating corolla tube venation, AN4 also regulates anther pigmentation $^{26}$. Similar to AN4, PeMYB12 determines not only venation patterning in sepals/petals, but also full pigmentation in the central lobe of the lip in Phalaenopsis spp. ${ }^{15}$. Chia et al. indicated that the dual functions of PeMYB12 may arise from a differential regulatory mechanism that exists in different floral organs ${ }^{15}$. In the MD line, the 
expression of AN4 was activated in its corolla tube but inactivated in its anthers (Fig. 2a), which indicates that $A N 4$ may be regulated via different mechanisms in the anther versus corolla tubes. The same expression pattern of AN4 was also observed in the V26 line. The inactivation of $A N 4$ in the anthers of V26 was believed to result from the methylation that occurred in the AN4 coding sequence and promoter, but the mechanism activating the expression of AN4 in the corolla tube remains unclear ${ }^{27}$. Additional efforts are therefore required to dissect the upstream regulatory mechanism of $A N 4$, which should be helpful for a better understanding of how AN4 activity is governed to form venation patterning.

In addition to $A N 4, V e 1$ also participates in the regulation of corolla tube venation patterning in petunia. Unlike the phenotype of the an 4 mutants, a few longitudinal pigmented stripes appeared in the corolla tube of $v e 1^{-}$individuals ${ }^{20}$, pointing to other as-of-yet unclear factors involved in determining corolla tube venation. Given the associations between $A N 4$ and Ve loci uncovered in genetic analysis ${ }^{20,25}$, elucidating the molecular nature of these $\mathrm{Ve}$ loci would provide insight into the mechanism underlying various venation patterns in petunia, making it a promising area of future research.

CRISPR/Cas9 is a powerful tool for creating null mutants and dissecting the functions of genes. To date, it has been used successfully to re-evaluate and redefine plant gene functions in a few cases ${ }^{39-41}$, from which novel insights were obtained. In the present work, the functioning of DPL and AN4 was re-evaluated in petunia using the CRISPR/Cas9 system. The obtained results strongly suggest that DPL regulates vein-associated anthocyanin patterning on the abaxial epidermis of flower buds, while AN4 determines corolla tube venation in petunia. These findings refine the regulatory framework of flower anthocyanin patterns in petunia and can facilitate further understanding of the mechanism underpinning these various patterns in petunias and perhaps other flowers.

\section{Materials and methods}

\section{Plant materials and growth conditions}

The petunia ( $P$. hybrida) inbred species MD was used in this study. Transgenic and MD plants were grown under greenhouse conditions ( $16 \mathrm{~h}$ light $/ 8 \mathrm{~h}$ dark photoperiod, $200-250 \mu \mathrm{mol} \mathrm{m} \mathrm{s}^{-1}$ light intensity, at $25^{\circ} \mathrm{C}$ ). For the cold treatment, the MD line and the $d p l-\mathrm{M} 3,-\mathrm{M} 4$, and -M5 mutants were cultured under greenhouse conditions until flowering, and the plants were placed in a growth chamber at $12 \pm 2{ }^{\circ} \mathrm{C}$ for 2 weeks with a $16 \mathrm{~h} \mathrm{light} / 8 \mathrm{~h}$ dark photoperiod and $120 \mu \mathrm{mol} \mathrm{m}^{-2} \mathrm{~s}^{-1}$ light intensity.

\section{Vector construction and transformation of petunia}

To isolate the AN4 cDNA from MD, the gene-specific primers $\mathrm{AN} 4^{\mathrm{MD}}-\mathrm{F} / \mathrm{R}$ were designed according to the sequence of $A N 4^{V 30}$ (accession number HQ428105). Total RNA extraction and cDNA synthesis were performed, as previously described ${ }^{42}$. The complete coding sequence of $A N 4^{M D}$ was isolated from the cDNA of MD petals. The PCR products were cloned into a pMD19-T vector (Takara, Dalian, China) and then sequenced by Tsingke Biological Technology (Chongqing, China). To overexpress $A N 4$ in $\mathrm{MD}$, the $p 35 S: A N 4$ vector was generated by cloning the coding sequence of $A N 4$ into a modified pGreenII0229 vector between the $35 \mathrm{~S}$ promoter and $35 \mathrm{~S}$ terminator. To generate $A N 4$-knockout mutants, the genomic sequences of $A N 4 I$ and $A N 4 I I$ were amplified from MD according to the genomic sequence of $P$. axillaris, and then, sgRNA targeting the border region of AN4 exons was designed using the CRISPR-GE web tool (skl. scau.edu.cn $)^{43}$. The sgRNA cassette was synthesized and cloned into a CRISPR/Cas9 construct to generate the pGGEAN4 vector, as described by Zhang et al. ${ }^{44}$. Likewise, to generate $D P L$-knockout mutants, a corresponding pGGEDPL vector was constructed as above. All the primers used for gene amplification or the construction of gene-editing vectors can be found in Supplementary Table 1. These recombinant vectors were introduced into Agrobacterium tumefaciens strain GV3101 by electroporation. The Agrobacterium-mediated transformation of petunia was performed according to Zhang et al. ${ }^{44}$.

\section{Detection of mutations}

Genomic DNA was first extracted from transgenic lines via the $C T A B$ method and then used as a template to amplify DNA fragments harboring the designed target sites with gene-specific primers (Supplementary Table 1). PCR was conducted using TopTaq DNA polymerase (Trans, Beijing, China) in a $25 \mu$ l volume. The amplification reaction was performed as follows: $94^{\circ} \mathrm{C}$ for $5 \mathrm{~min}$, followed by $28-30$ cycles of $94{ }^{\circ} \mathrm{C}$ for $30 \mathrm{~s}, 55^{\circ} \mathrm{C}$ for $30 \mathrm{~s}$, and $72{ }^{\circ} \mathrm{C}$ for $2 \mathrm{~min}$, and then $72{ }^{\circ} \mathrm{C}$ for $10 \mathrm{~min}$. The PCR products were separated by $1 \%$ agarose gel electrophoresis and directly sequenced by Sanger sequencing. Then, the sequencing chromatograms were decoded into allelic sequences to identify the mutations using the CRISPR-GE web tool (skl.scau.edu.cn) ${ }^{45}$. The sequencing chromatograms are presented in Fig. S3.

\section{Real-time PCR analysis}

Total RNA extraction and cDNA synthesis were carried out as previously described ${ }^{42}$. Quantitative real-time PCR was performed with Ssofast EvaGreen Supermix (Bio-Rad, Hercules, CA, USA) on a CFX96 Real-time PCR System (Bio-Rad, Hercules, CA, USA). Primers for anthocyanin biosynthetic genes were designed according to Albert et al. ${ }^{34}$. The $S A N D$ gene was used to normalize the samples $^{46}$. Relative gene expression values were calculated using the methodology of Schmittgen and Livak ${ }^{47}$. The 
qPCR data are presented here as the mean $\pm \mathrm{SE}$ of three biological replicates. All primer sequences used for qPCR are listed in Supplementary Table 1.

\section{Anthocyanin measurement}

Extraction of anthocyanins was performed, as described by Viola et al. ${ }^{48}$. Briefly, $0.15 \mathrm{~g}$ fresh samples were ground into powder using liquid nitrogen and then incubated in $15 \mathrm{~mL}$ of $1 \% \mathrm{HCl}$-methanol $(\mathrm{v} / \mathrm{v})$ at $4{ }^{\circ} \mathrm{C}$ overnight in the dark. After centrifuging each sample, the absorbance of the resulting supernatant was measured at 530 and $657 \mathrm{~nm}$ on a Varioskan Flash Spetral Scanning Multimode Reader (Thermo Fisher, Waltham, MA, USA). The relative anthocyanin content was then determined as $\left(A_{530}-\right.$ $\left.0.25 \times A_{657}\right)$ per gram fresh weight.

\section{Statistical analysis}

The significance of differences among the data were determined by Student's $t$ test using SPSS 17.0 software. Statistically significant differences are indicated by asterisks * $(P<0.05)$ or $* *(P<0.01)$.

\section{Accession numbers}

The sequences of the genes described in this work can be found in GenBank: DPL, HQ116169; AN4, HQ428104. The genome sequences of AN4I and AN4II can be obtained from the Sol Genomic Network (https://solgenomics.net/) under the accession numbers Peaxi162Scf00578g00008.1 and Peaxi162Scf00578g00007.1, respectively.

\section{Acknowledgements}

This work was supported by the National Natural Science of China (31272199) and Fundamental Research Funds for the Central Universities (XDJK2020D038).

\section{Author details \\ ${ }^{1}$ Chongqing Engineering Research Center for Floriculture, Key Laboratory of Horticulture Science for Southern Mountainous Regions, Ministry of Education, College of Horticulture and Landscape Architecture, Southwest University, 400716 Chongqing, China. ${ }^{2}$ College of Agriculture, Guizhou University, 550025 Guiyang, Guizhou, China. ${ }^{3}$ Sichuan Provincial Key Laboratory for Development and Utilization of Characteristic Horticultural Biological Resources, College of Chemistry and Life Sciences, Chengdu Normal University, 611130 Chengdu, China}

\section{Author contributions}

Y.G. designed the experiments. B.Z., X.X., and S.Y. performed the experiments. B.Z. and R.H. analyzed the data. M.L. and Y.G. contributed materials and reagents. Y.G. and B.Z. wrote and edited the manuscript.

\section{Conflict of interest}

The authors declare no competing interests.

Supplementary information The online version contains supplementary material available at https://doi.org/10.1038/s41438-021-00555-6.

Received: 10 November 2020 Revised: 25 March 2021 Accepted: 1 April 2021

Published online: 01 June 2021

\section{References}

1. Grotewold, E. The genetics and biochemistry of floral pigments. Annu. Rev. Plant Biol. 57, 761-780 (2006).

2. Quattrocchio, F., Wing, J. F., Leppen, H., Mol, J. \& Koes, R. E. Regulatory genes controlling anthocyanin pigmentation are functionally conserved among plant species and have distinct sets of target genes. Plant Cell 5, 1497-1512 (1993).

3. Kubasek, W. L. et al. Regulation of flavonoid biosynthetic genes in germinating arabidopsis seedlings. Plant Cell 4, 1229-1236 (1992).

4. Martin, C., Prescott, A., Mackay, S., Bartlett, J. \& Vrijlandt, E. Control of anthocyanin biosynthesis in flowers of Antirrhinum majus. Plant J. 1, 37-49 (1991).

5. Zhang, Y., Butelli, E. \& Martin, C. Engineering anthocyanin biosynthesis in plants. Curr. Opin. Plant Biol. 19, 81-90 (2014).

6. Lai, Y., Li, H. \& Yamagishi, M. A review of target gene specificity of flavonoid R2R3-MYB transcription factors and a discussion of factors contributing to the target gene selectivity. Front. Biol. 8, 577-598 (2013).

7. Petroni, K. \& Tonelli, C. Recent advances on the regulation of anthocyanin synthesis in reproductive organs. Plant Sci. 181, 219-229 (2011).

8. Gao, Y. et al. Tomato SIAN11 regulates flavonoid biosynthesis and seed dormancy by interaction with bHLH proteins but not with MYB proteins. Hortic. Res. 5, 27 (2018).

9. Sun, B. et al. Purple foliage coloration in tea (Camellia sinensis L.) arises from activation of the R2R3-MYB transcription factor CsAN1. Sci. Rep. 6, 32534 (2016).

10. Yamagishi, M. A novel R2R3-MYB transcription factor regulates light-mediated floral and vegetative anthocyanin pigmentation patterns in Lilium regale. Mol. Breeding 36, 3 (2016).

11. Xu, Z. S., Yang, Q. Q., Feng, K., Yu, X. \& Xiong, A. S. DcMYB113, a root-specific R2R3-MYB, conditions anthocyanin biosynthesis and modification in carrot. Plant Biotechnol. J. 18, 1585-1597 (2020).

12. Davies, K. M., Albert, N. W. \& Schwinn, K. E. From landing lights to mimicry: the molecular regulation of flower colouration and mechanisms for pigmentation patterning. Funct. Plant Biol. 39, 619-638 (2012).

13. Shang, Y. J. et al. The molecular basis for venation patterning of pigmentation and its effect on pollinator attraction in flowers of Antirrhinum. N. Phytologist 189, 602-615 (2011).

14. Schwinn, K. et al. A small family of MYB-regulatory genes controls floral pigmentation intensity and patterning in the genus Antirrhinum. Plant Cell 18, 831-851 (2006)

15. Hsu, C. C., Chen, Y. Y., Tsai, W. C., Chen, W. H. \& Chen, H. H. Three R2R3-MYB transcription factors regulate distinct floral pigmentation patterning in Phalaenopsis spp. Plant Physiol. 168, 175-191 (2015).

16. Gu, Z. et al. A novel R2R3-MYB transcription factor contributes to petal blotch formation by regulating organ-specific expression of PsCHS in tree peony (Paeonia suffruticosa). Plant Cell Physiol. 60, 599-611 (2019).

17. Yamagishi, M., Shimoyamada, Y., Nakatsuka, T. \& Masuda, K. Two R2R3-MYB genes, homologs of Petunia AN2, regulate anthocyanin biosyntheses in flower Tepals, tepal spots and leaves of asiatic hybrid lily. Plant Cell Physiol. 51, 463-474 (2010).

18. Ding, B. et al. Two MYB proteins in a self-organizing activator-inhibitor system produce spotted pigmentation patterns. Curr. Biol. 30, 802-814 (2020).

19. Bombarely, A. et al. Insight into the evolution of the Solanaceae from the parental genomes of Petunia hybrida. Nat. Plants 2, 16074 (2016).

20. Wiering, H. d. V. P. In Monographs on Theoretical and Applied Genetics 9: Petunia (ed Sink, K. C.) 49-76 (Springer, 1984).

21. Albert, N. W. et al. Members of an R2R3-MYB transcription factor family in Petunia are developmentally and environmentally regulated to control complex floral and vegetative pigmentation patterning. Plant J. 65, 771-784 (2011).

22. Yuan, Y. W., Rebocho, A. B., Sagawa, J. M., Stanley, L. E. \& Bradshaw, H. D. Competition between anthocyanin and flavonol biosynthesis produces spatial pattern variation of floral pigments between Mimulus species. Proc. Natl Acad. Sci. USA 113, 2448-2453 (2016).

23. Zhang, $H$. et al. Identification and functional analysis of three new anthocyanin R2R3-MYB genes in Petunia. Plant Direct 3, e00114 (2019).

24. Yamagishi, M., Toda, S. \& Tasaki, K. The novel allele of the LhMYB12 gene is involved in splatter-type spot formation on the flower tepals of Asiatic hybrid lilies (Lilium spp.). N. Phytologist 201, 1009-1020 (2014).

25. Janick, J. Plant Breeding Reviews, Vol. 30 (Wiley, 2008). 
26. Quattrocchio, F., Wing, J. F., Leppen, H. T. C., Mol, J. N. M. \& Koes, R. E. Regulatory genes-controlling anthocyanin pigmentation are functionally conserved among plant-species and have distinct sets of target genes. Plant Cell 5, 1497-1512 (1993).

27. Povero, G. Physiological and Genetic Control of Anthocyanin Pigmentation in Different Species. PhD thesis (2011).

28. Albert, N. W. et al. Light-induced vegetative anthocyanin pigmentation in Petunia. J. Exp. Bot. 60, 2191-2202 (2009).

29. Ai, T. N., Arun, M., Naing, A. H., Lim, S. H. \& Kim, C. K. Combinatorial expression of transcription factor genes B-Peru and MPAP1 enhances anthocyanin accumulation in transgenic Petunia hybrid. Sci. Hortic. 200, 186-196 (2016).

30. Schwinn, K. E. et al. MYB and bHLH transcription factor transgenes increase anthocyanin pigmentation in petunia and lisianthus plants, and the petunia phenotypes are strongly enhanced under field conditions. Front. Plant Sci. 5, 603 (2014).

31. van Houwelingen, A., Souer, E., Mol, J. \& Koes, R. Epigenetic interactions among three dTph1 transposons in two homologous chromosomes activate a new excision-repair mechanism in petunia. Plant Cell 11, 1319-1336 (1999).

32. Koseki, M., Goto, K., Masuta, C. \& Kanazawa, A. The star-type color pattern in Petunia hybrida 'Red star' flowers is induced by sequence-specific degradation of chalcone synthase RNA. Plant Cell Physiol. 46, 1879-1883 (2005).

33. Saito, R. et al. Regulation of anthocyanin biosynthesis involved in the formation of marginal picotee petals in Petunia. Plant Sci. 170, 828-834 (2006).

34. Albert, N. W. et al. A conserved network of transcriptional activators and repressors regulates anthocyanin pigmentation in eudicots. Plant Cell 26, 962-980 (2014).

35. Quattrocchio, F. et al. Molecular analysis of the anthocyanin2 gene of petunia and its role in the evolution of flower color. Plant Cell 11, 1433-1444 (1999).

36. Quattrocchio, F., Wing, J. F., van der Woude, K., Mol, J. N. M. \& Koes, R. Analysis of bHLH and MYB domain proteins: species-specific regulatory differences are caused by divergent evolution of target anthocyanin genes. Plant J. 13 475-488 (1998).
37. Stracke, R. et al. Differential regulation of closely related R2R3-MYB transcription factors controls flavonol accumulation in different parts of the Arabidopsis thaliana seedling. Plant J. Cell Mol. Biol. 50, 660-677 (2007).

38. Dubos, C. et al. MYB transcription factors in Arabidopsis. Trends Plant Sci. 15 573-581 (2010).

39. Ito, $Y$. et al. Re-evaluation of the rin mutation and the role of RIN in the induction of tomato ripening. Nat. Plants 3, 866-874 (2017).

40. Dell'Aglio, E., Dalvit, I., Loubery, S. \& Fitzpatrick, T. B. Clarification of the dispensability of PDX1.2 for Arabidopsis viability using CRISPR/Cas9. BMC Plant Biol. 19, 464 (2019).

41. Yan, S. et al. Anthocyanin Fruit encodes an R2R3-MYB transcription factor, SIAN2-like, activating the transcription of SIMYBATV to fine-tune anthocyanin content in tomato fruit. N. Phytol. 225, 2048-2063 (2020).

42. Guo, Y. L. et al. Undesired small RNAs originate from an artificial microRNA precursor in transgenic Petunia (Petunia hybrida). PLoS ONE 9, e98783 (2014).

43. Xie, X. R. et al. CRISPR-GE: a convenient software toolkit for CRISPR-based genome editing. Mol. Plant 10, 1246-1249 (2017).

44. Zhang, B., Yang, X., Yang, C. P., Li, M. Y. \& Guo, Y. L. Exploiting the CRISPR/ Cas9 system for targeted genome mutagenesis in Petunia. Sci. Rep. 6, 20315 (2016).

45. Liu, W. Z. et al. DSDecode: a web-based tool for decoding of sequencing chromatograms for genotyping of targeted mutations. Mol. plant $\mathbf{8}$, 1431-1433 (2015).

46. Mallona, I., Lischewski, S., Weiss, J., Hause, B. \& Egea-Cortines, M. Validation of reference genes for quantitative real-time PCR during leaf and flower development in Petunia hybrida. BMC Plant Biol. 10, 4 (2010).

47. Schmittgen, T. D. \& Livak, K. J. Analyzing real-time PCR data by the comparative C-T method. Nat. Protoc. 3, 1101-1108 (2008).

48. Viola, I. L., Camoirano, A. \& Gonzalez, D. H. Redox-dependent modulation of anthocyanin biosynthesis by the TCP transcription factor TCP15 during exposure to high light intensity conditions in Arabidopsis. Plant Physiol. 170, 74-85 (2016). 